\title{
Differential photocurrent spectroscopy: A novel technique for semiconductor characterization
}

\author{
Richard L. Tober \\ Harry Diamond Laboratories, U.S. Army Adelphi Laboratory Center. 2800 Powder Mill Road, \\ Adelphi, Maryland 20783-1197 \\ W. Q. Li and P. K. Bhattacharya \\ Department of Electrical Engineering and Computer Science, University of Michigan, \\ Ann Arbor, Michigan 48109-2122 \\ (Received 20 September 1991; accepted for publication 2 January 1992) \\ Differential photocurrent spectra of various $\operatorname{In}_{x} \mathrm{Ga}_{1}-x$ As diodes demonstrate that modulated \\ photocurrent techniques can easily be used to characterize $p-i-n$ and Schottky diode \\ structures. The spectra were obtained in such a way as to produce a first derivative with \\ respect to either the photon energy or an electric field. A simple model yields an expression for \\ the differential photocurrent spectra in terms of the derivative functional form normally \\ used to fit modulated spectra. Therefore, the sharp spectral features produced with these \\ techniques can be used to determine not only band-gap energies and spectral linewidths \\ but built-in electric fields as well.
}

\section{INTRODUCTION}

Within the last 25 years numerous optical modulation techniques have evolved, each with particular advantages and circumstances for which they are suited. ${ }^{1-6}$ In each case, straightforward methods exist to fit spectral data in order to extract information about transition energies, linewidths, or even internal electric fields. However, specific device structures (e.g., waveguides and mesa diodes) have geometrical constraints that inhibit most optical modulation techniques. This compelled us to develop techniques with which to use photocurrent measurements to characterize device structures and thus supplement the other modulation spectroscopies mentioned above.

In this communication, we describe a novel way to use photocurrent spectra to characterize layered structures having $p-i-n$ or Schottky diode configurations. The differential photocurrent (DPC) spectra obtained as shown below can be fit with the same derivative functional form normally used with techniques such as photoreflectance $(P R)$ or piezoreflectance $(\mathrm{PzR}) .^{1}$ We justify using this functional form by relating the lth-order differential of photocurrent spectra to the lth-order differential of the dielectric function. As proof of principle, we present spectral data obtained in such a fashion to produce spectra for InGaAs diodes either resulting from a first derivative with respect to the photon energy or an electric field. In the former case, transition energies extracted from the spectra specified the indium concentration of our samples. The latter spectra had Franz-Keldysh oscillations with which we determined the magnitude of internal electric fields.

\section{THEORY}

We neglect the effects of multiple reflections and consider that absorption occurring within the depletion region of either a $p-i-n$ or Schottky diode. It has been shown that dips in photocurrent spectra can occur if carriers recombine before being collected in the $n$ or $p$ regions. $^{7}$ There- fore, we also assume that the electric field is strong enough so that carrier lifetimes do not affect the magnitude of the photocurrent. The photocurrent can be written as ${ }^{8}$

$$
i_{\mathrm{ph}}=I_{0} \eta \frac{e}{\hbar \omega}(T)\left(1-e^{-\alpha x}\right),
$$

where $\eta$ is the quantum efficiency, $T$ represents the power transmission coefficient, and $I_{0}$ is the intensity incident on the interface defining the active region, and $1-e^{-\alpha x}$ is the fractional power absorbed in the layer of width $x$. The differential of Eq. (1) is

$$
\Delta i_{\mathrm{ph}}=I_{0} \eta \frac{e}{\hbar \omega}\left[T x \Delta \alpha e^{-\alpha x}+\left(1-e^{-\alpha x}\right) \Delta T\right] .
$$

The differential of the transmission coefficient, $\Delta T$, is in general a function of the complex indices of refraction of the regions on either side of the interface. However, we consider contributions only from the active region, assume normal incidence, and write

$$
\Delta T=\frac{\partial T}{\partial n} \Delta n+\frac{\partial T}{\partial k} \Delta k
$$

The change in the real and imaginary parts of the index of refraction can be written as functions of the corresponding changes in the complex diclcetric function. Therefore, after collecting constants and replacing $\alpha$ with $2 \pi k / \lambda$, we can write Eq. (2) as

$$
\Delta i_{\mathrm{ph}}=\sum_{i=1}^{N} \operatorname{Re}(A+i B) \Delta \widetilde{\epsilon},
$$

where $A$ and $B$ are similar to the Seraphin coefficients ${ }^{9}$ in that they are functions of the unperturbed complex indexes but differ from them because of their dependence on layer thicknesses.

The complex dielectric function of a layer can be written as ${ }^{1}$ 


$$
\tilde{\epsilon}-1=B e^{i \theta}(\Delta E+i \Gamma)^{m},
$$

where $\theta$ is a phase angle that depends on the magnitudes of the real and imaginary parts of the dielectric function, $\Delta E$ is the transition energy, $m$ depends on the dimensionality of the joint density of states, and $\Gamma$ is the phenomenological broadening parameter. Since $\Delta \widetilde{\epsilon}$ can be expressed as either a first or third derivative of the unperturbed dielectric function, ${ }^{1}$ an $l$ th-order derivative of Eq. (5) can be used in Eq. (4) so that, in general, the modulated photocurrent can be expressed as

$\Delta i_{\mathrm{ph}}=\sum_{j=1}^{M} \frac{C_{j} \cos \left[\theta_{j}+d_{j} \cos { }^{-1} \sqrt{\Delta E_{j}^{2} /\left(\Delta E_{j}^{2}+\Gamma_{j}^{2}\right)}\right]}{\left(\Delta E_{j}^{2}+\Gamma_{j}^{2}\right)^{d_{j} / 2}}$,

where $M$ is an index associated with each critical point, $C_{j}$ is the amplitude of the jth critical point and is proportional to the differential with respect to the perturbing parameter, $d_{j}=l_{j}-m_{j}$, and $l_{j}$ is the order of the derivative. Spectral data fits to Eq. (6) yield information about transition energies, broadening parameters, and the dimensionality of the dielectric function of the material probed.

\section{EXPERIMENTAL CONSIDERATIONS}

Photocurrent measurements were made on InGaAs/ GaAs $p-i-n$ diode structures grown on $n^{+}$[100] GaAs substrates. The $1-\mu \mathrm{m}$ InGaAs intrinsic regions were wider than the critical thickness and thus strain free. ${ }^{10}$ The $p^{+}$ layers were $1000 \AA$ thick and had the same indium concentrations as the intrinsic regions. The group three fluxes werc set during molecular-beam epitaxy (MBE) growth so that nominal indium concentrations of samples 1523,1524 , and 1525 would be $5 \%, 10 \%$, and $15 \%$, respectively. The diodes had $100-\AA$ semitransparent gold electrodes deposited on the $p^{+}$surface and 1000- $\AA$ electrodes on the $n^{+}$ back surface.

Optically chopped $(400 \mathrm{~Hz})$ radiation from a tungsten-halogen lamp was dispersed through a $\frac{2}{3}-\mathrm{m}$ monochromator and focused onto samples which were connected to a current-voltage converter. A lock-in amplifier tuned to the chopping frequency measured the output voltage of the $I-V$ converter. Differential spectra were obtained by either digitally calculating the first derivative of the (room-temperature) photocurrent spectra with respect to energy or subtracting ( $78 \mathrm{~K}$ ) spectra obtained with different applied biases. We did not normalize the spectra to eliminate their dependence on the incident intensity since the radiation from the tungsten lamp was approximately constant over the narrow spectral ranges used for each spectrum. (We do, however, acknowledge that care should be exercised when making this approximation, especially when using a source such as a xenon arc lamp.)

\section{RESULTS}

We could not easily ascribe transition energies to the broad unmodulated spectra of the InGaAs diode structures shown in Fig. 1(a), nor could we use the extrema in the differential photocurrent spectra shown if Fig. 1(b) with-

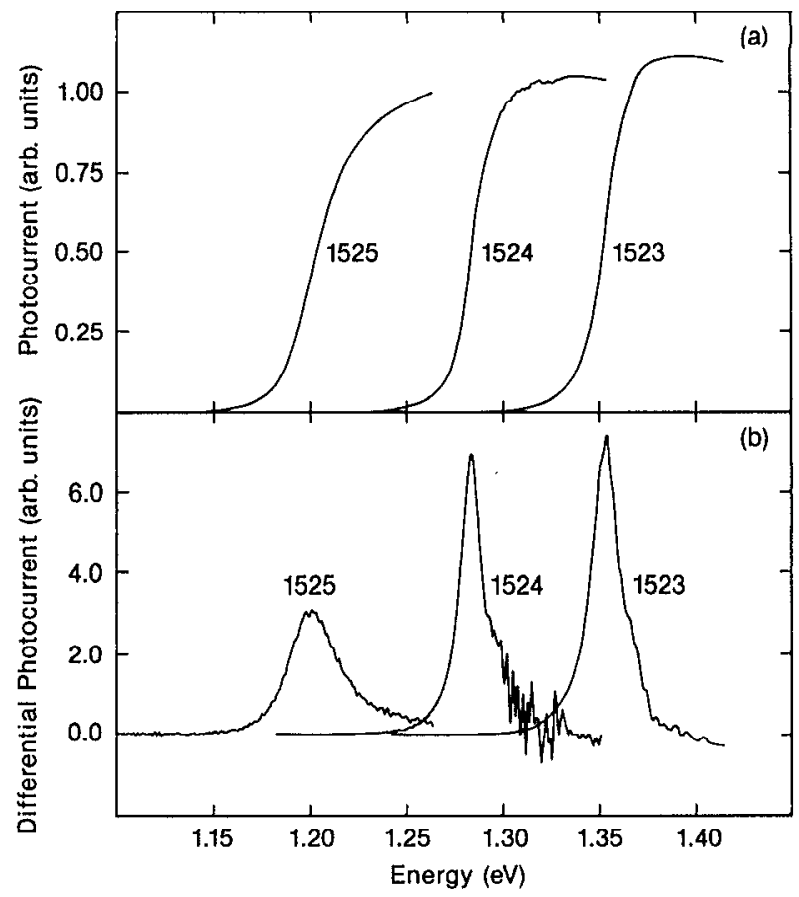

FIG. 1. (a) Normalized photocurrent spectra obtained at $295 \mathrm{~K}$ of the three InGaAs $p-i-n$ diode structures; (b) first derivative of photocurrent spectra with respect to photon energy.

out justification. Consequently, we compared transition energies obtained from both PzR and DPC spectra using Eq. (6) and parameters appropriate for the first derivative of three-dimensional critical points. This was reasonable since previous work has shown that fitting PzR spectra to Eq.

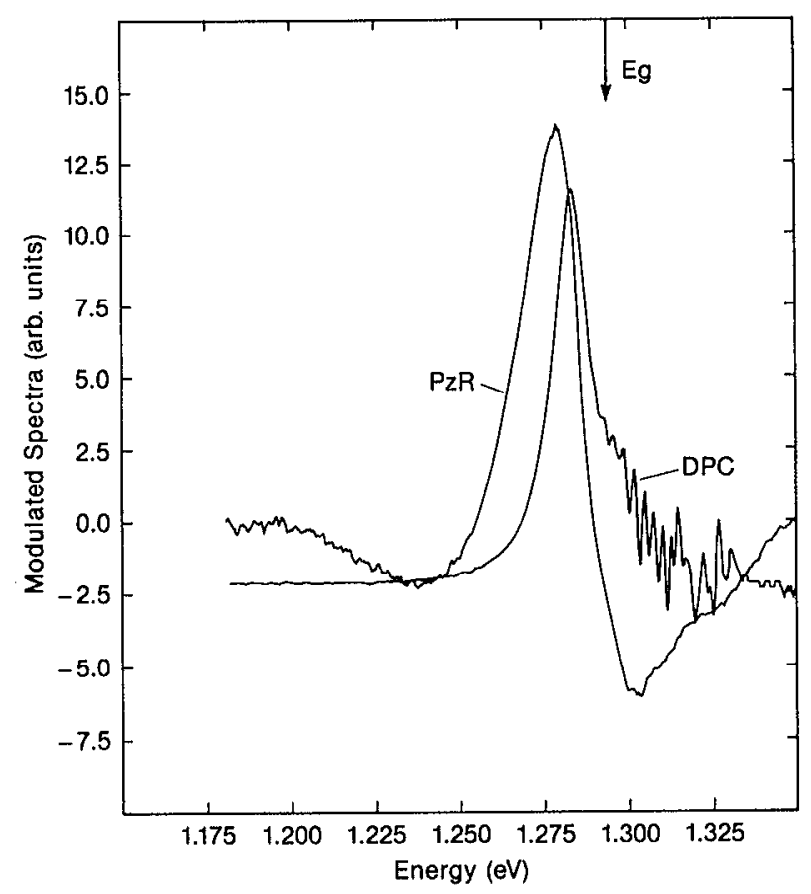

FIG. 2. Comparison of PzR and DPD spectra of sample 1524; fits of both spectra yield transition energies of $1.284 \mathrm{eV}$. 
TABLE I. The DPC transition energies for the InGaAs $p-i-n$ diode structures, and their calculated ${ }^{\mathrm{a}}$ and nominal indium concentrations.

\begin{tabular}{llcc}
\hline \hline & $E_{\boldsymbol{g}}$ & \multicolumn{2}{c}{ Percent Indium } \\
\cline { 3 - 4 } Sample & $\left(\mathrm{eV}^{\mathrm{b}}\right)^{\mathrm{b}}$ & Nominal & Experiment \\
\hline 1523 & 1.355 & 5 & 4.4 \\
1524 & 1.284 & 10 & 9.1 \\
1525 & $1.284^{\mathrm{c}}$ & & \\
\hline
\end{tabular}

Determined using $E_{8}=0.36+0.55(1-x)+0.515(1-x)^{2}$.

${ }^{b} \pm 0.002 \mathrm{eV}$.

${ }^{c}$ From PzR measurements.

(6) is a viable means to determine transition energies. ${ }^{11}$ The values thus obtained agreed, but we also noted that the band-gap energy indicated with an arrow in Fig. 2 did not correspond to the extrema of the DPC spectrum. Having gained confidence in differential photocurrent spectra, we extracted [using Eq. (6)] transition energies from the remaining spectra of Fig. 1(b) and calculated the corresponding indium concentrations. Table I lists the transition energies and the nominal and experimental indium concentrations for the samples discussed.

The fits to the experimental data obtained from the patterned samples (see Fig. 3) were reasonable within at least $3 \Gamma$ of the transition energies. The additional structure on the high-energy side of the extrema in the spectra of samples 1523 and 1524 was repeatable. The agreement between the transition energies extracted from the $P z R$ and DPC spectra of sample 1524 (Fig. 2), however, indicates that this high-energy structure was inconsequential for determining alloy concentrations. We defer an explanation of this structure until it is better understood and only suggest that it results from electric field effects near the InGaAs/ GaAs heterojunction. ${ }^{12}$

We also obtained photocurrent and PzR spectra of the In GaAs diode samples at $78 \mathrm{~K}$ to minimize thermal broadening and enhance effects due to built-in electric fields. Figure 4(a) shows two photocurrent spectra of sample

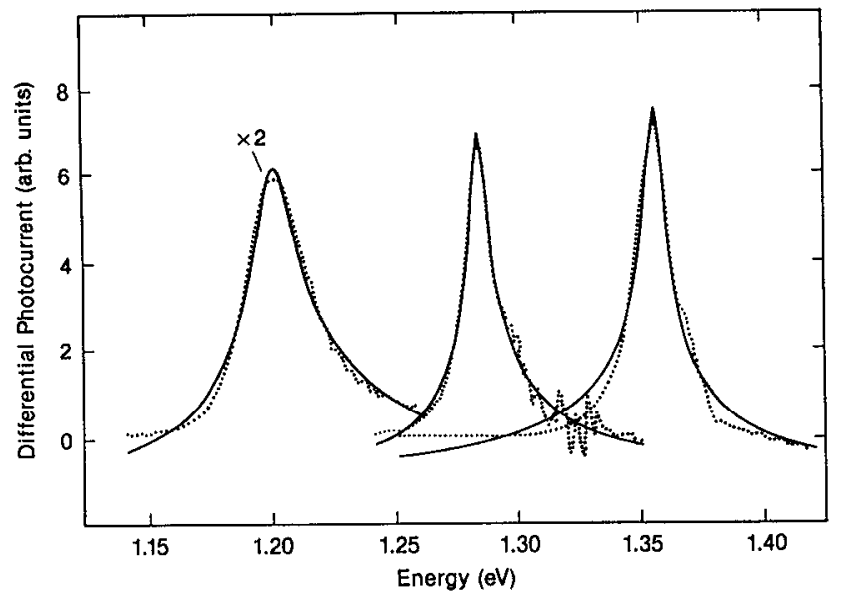

FIG. 3. Experimental data (dots) and fit (solid) of the differential photocurrent spectra.

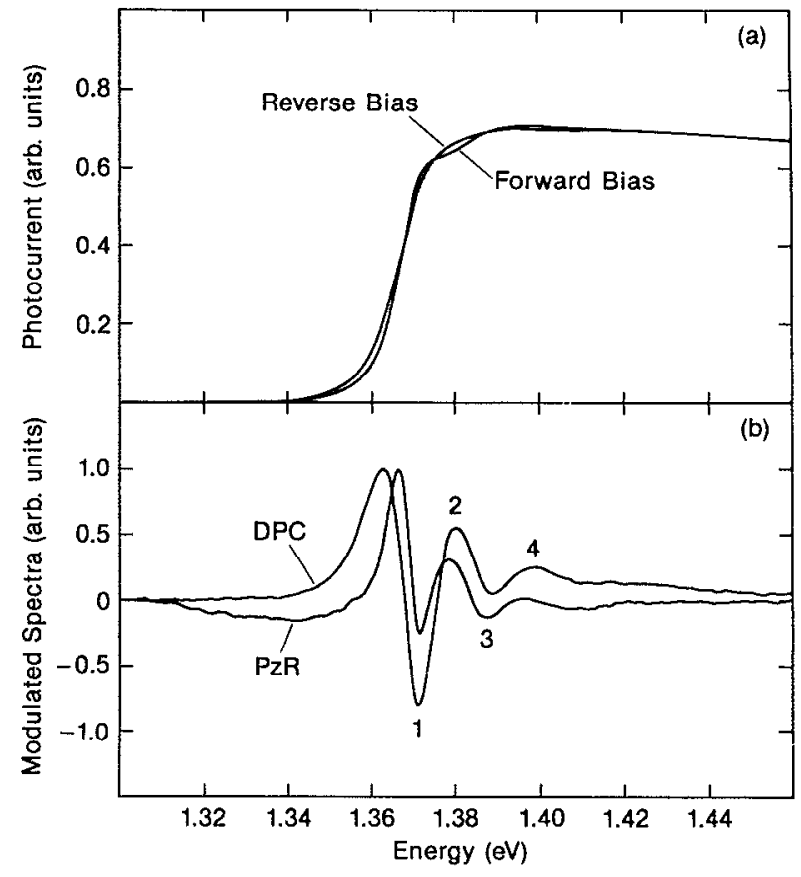

FIG. 4. (a) Photocurrent spectra obtained at $78 \mathrm{~K}$ of sample 1524 with forward and reverse biases. (b) Differential photocurrent spectra [calculated by subtracting the two spectra in (a)] and a piezoreflectance spectrum of the unbiased sample.

1524 , one obtained with a $0.28-\mathrm{V}$ reverse bias (increasing the built-in field by $\sim 2.8 \mathrm{kV} / \mathrm{cm}$ as determined from growth parameters) and the other with a $0.1-\mathrm{V}$ forward bias (decreasing the built-in field by $\sim 1 \mathrm{kV} / \mathrm{cm}$ ). By subtracting the latter from the former spectrum, we produced the DPC spectrum in Fig. 4(b) and used the FranzKeldysh oscillations to determine the maximum field (i.e., built-in plus reverse-bias field) to be $10 \mathrm{kV} / \mathrm{cm}$. (This interpretation of the electric field follows directly from discussions by Bhattacharya et $a l^{2}$ ) The PzR spectrum shown in Fig. 4(b) was obtained without an applied bias, its Franz-Keldysh oscillations corresponding to a field of $\sim 7.5 \mathrm{kV} / \mathrm{cm}$. The differences between the electric fields determined from the spectra of Fig. 4(b) were consistent with the magnitudes of the applied and internal electric fields. This suggested that differential photocurrent spectra can also be used for Franz-Keldysh analysis of semiconductors.

\section{SUMMARY}

We have shown that differential photocurrent spectroscopy provides a valuable means to characterize layered semiconductor structures. Spectra can be fit with the derivative functional form normally used in conjunction with other differential techniques such as PzR, PR, and electroreflectance (ER). Transition energies thus extracted from the differential photocurrent spectra agree well within experimental error with those obtained from piezoreflectance spectra. Moreover, DPC spectra can be used for Franz-Keldysh analysis of semiconductors. Thus informa- 
tion regarding transition energies, broadening parameters, dimensionality, effective masses, and built-in fields can be easily acquired using this versatile technique.

${ }^{1}$ D. E. Aspnes, in Handbook on Semiconductors, edited by T. S. Moss (North-Holland, Amsterdam, 1980), Vol. 2, p. 109.

${ }^{2}$ R. N. Bhattacharya, H. Shen, P. Parayyanthal, F. Pollak, T. Couts, and II. Aharoni, Phys. Rev. B 37, 4044 (1988).

${ }^{3}$ R. L. Tober, J. Pamulapati, P. K. Bhattacharya, and J. E. Oh, J. Electron. Mater. 18, 379 (1989).

${ }^{4}$ M. Garfinkel, J. J. Tieman, and W. E. Engeler, Phys. Rev. 148, 695 (1966).

${ }^{5}$ R. L. Tober, J. P. Ferraris, and R. Glosser, Phys. Rev, B 33, 8768 (1986).

${ }^{6}$ R. E. Hummel, Proceedings of the Society of Photo-Optical Instrumen- tation Engineers, San Diego, 1990, edited by F. H. Pollak (SPIE, Bellingham, WA, 1990), Vol. 1286, p. 146.

${ }^{7}$ R. P. Leavitt and J. L. Bradshaw, Appl. Phys. Lett. 59, 2433 (1991).

${ }^{8}$ S. M. Sze, Physics of Semiconductor Devices, 2nd ed. (Wiley, New York, 1981), pp. 800-805.

${ }^{9}$ B. O. Seraphin and N. Bottka, Phys. Rev. 145, 628 (1966).

${ }^{10}$ W. Q. Li, P. K. Bhattacharya, and R. L. Tober, Appl. Phys. Lett. 58, 1931 (1991).

${ }^{11}$ It has been our experience that fits of PzR, ER, and PR spectra to Eq. (6) yield the same transition energies for spectra obtained from not only quantum-well samples but bulk GaAs and AlGaAs as well. See, for examples, Ref. 3; R. L. Tober, A. L. Smirl, T. F. Boggess, and J. N. Schulman, J. Appl. Phys. 64, 4678 (1988), and references cited therein for specifics regarding fitting parameters.

${ }^{12}$ R. L. Tober (unpublished). 